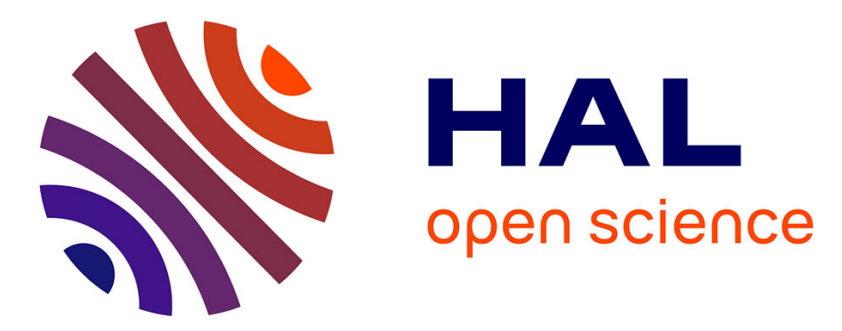

\title{
Sciences et société en interaction sur Internet. Éléments pour une histoire de l'édition électronique en sciences humaines et sociales
}

Pierre Mounier, Marin Dacos

\section{- To cite this version:}

Pierre Mounier, Marin Dacos. Sciences et société en interaction sur Internet. Éléments pour une histoire de l'édition électronique en sciences humaines et sociales. Communication \& langages, 2009, 159, pp.123-135. sic_00439828

\section{HAL Id: sic 00439828 \\ https://archivesic.ccsd.cnrs.fr/sic_00439828}

Submitted on 8 Dec 2009

HAL is a multi-disciplinary open access archive for the deposit and dissemination of scientific research documents, whether they are published or not. The documents may come from teaching and research institutions in France or abroad, or from public or private research centers.
L'archive ouverte pluridisciplinaire HAL, est destinée au dépôt et à la diffusion de documents scientifiques de niveau recherche, publiés ou non, émanant des établissements d'enseignement et de recherche français ou étrangers, des laboratoires publics ou privés. 


\section{Sciences et société en interaction sur Internet ${ }^{1}$}

Eléments pour une histoire de l'édition électronique en sciences humaines et sociales

Marin Dacos et Pierre Mounier

Résumé : Dans l'histoire des rapports complexes qu'entretiennent sciences et société, le développement des réseaux numériques constitue un moment stratégique, que ce soit au niveau de leur développement technique, ou des modifications que ce développement produit sur les formes de la communication scientifique. Le cas particulier des sciences humaines et sociales met bien en évidence le rôle de médiation que les TIC jouent dans les relations entre sciences et société.

Mots clés : Internet, sciences, communication, société, édition, édition électronique, archives ouvertes, XML, blogs, revues

Ce qui caractérise l'usage des TIC dans les pratiques de communication en sciences humaines et sociales, c'est, nous semble-t-il, qu'elles réactivent d'une manière particulière la tension que ces disciplines connaissent traditionnellement entre un pôle de scientificité, où les sciences de la nature jouent un rôle prédominant, et un espace public de plus en plus problématisé par l'intermédiaire de la notion de « société de l'information ». Il s'agit donc d'abord de comprendre comment les TIC se développent au coeur d'une relation sciences-société en reconfiguration, comme un vecteur parmi d'autres du renforcement d'un cadre global de "scientifisation» des activités sociales. D'une certaine manière, le développement de l'usage des TIC en sciences humaines et sociales, est parfois accompagné d'un durcissement des critères de scientificité dans les pratiques de recherche d'un grand nombre de disciplines. Pour autant, il serait faux de ne voir dans les actuels usages des TIC dans ces disciplines, qu'un assujettissement de leurs pratiques de recherche et de publication, comme certains acteurs du milieu l'interprètent quelquefois. Un examen attentif du foisonnement actuel des initiatives d'édition électronique montre au contraire que l'invention de nouvelles formes de publication en sciences humaines et sociales s'alimente aussi, et par effet de retour, aux nouvelles formes de circulation de l'information que l'on voit se développer hors du contexte scientifique.

Les représentations de sens commun à propos de la question de la « publicisation » des sciences, ou appelée autrement selon les époques la vulgarisation ou la médiation scientifique, postulent la préexistence de deux instances bien séparées, les sciences d'un côté, la société de l'autre, campées sur les deux rives d'un fossé d'incompréhension qui les sépare, en travers duquel l'activité de publicisation tente de jeter des ponts par des moyens plus ou moins sophistiqués ou technicisés. C'est cette approche que les plus récentes recherches sur le sujet ont pu remettre en cause. On a récemment montré [SCH 05] en effet, combien les grandes évolutions dans les pratiques de «mise en public des sciences », ne manifestent pas tant une hypothétique plus grande efficacité des modes de communication adoptés, qu'une redéfinition du rapport sciences-société, souvent provoquée par des évolutions internes au champ scientifique. Cette redéfinition, fait remarquer Schiele, prend d'ailleurs la forme d'une reterritorialisation, qui déplace les lieux de prise de parole. Les revues, les musées de sciences, les centres de culture scientifique et technique, les médias audio-visuels, sont autant de lieux où s'opèrent ces déplacements à différents moments stratégiques de la recomposition des relations science-société. C'est dans cette perspective qu'il faut donc inscrire toute interrogation sur la manière dont les technologies de l'information et de la communication (TIC) peuvent constituer une instance de médiation où s'opère une actualisation particulière de l'activité de

Ce texte devait initialement paraître dans l'ouvrage Humanités Numériques, publié aux éditions Hermes Lavoisier, sous la direction de Bernard Reber et Claire Brossaud. Les conditions imposées par l'éditeur ne nous autorisaient cependant pas à en déposer copie sur une archive ouverte comme HAL SHS. C'est pourquoi nous avons retiré notre proposition. Nous remercions vivement la revue Communication et Langages d'avoir accepté d'accueillir notre contribution. 
publicisation des sciences.

\section{Des technologies pour la communication scientifique}

Du strict point de vue de la chronologie, le développement des technologies de l'information et de la communication correspond tout à la fois à une intensification sans précédent de l'activité de communication scientifique dans tous les domaines, en même temps qu'à une rationalisation de l'organisation de la recherche qui prend, dans tous les pays développés, une dimension industrielle, en grande partie d'ailleurs sous l'impulsion de l'Etat. L'article fondateur de Vannevar Bush, « As we may think », publié dans la revue The Atlantic [BUS 45], qui tire les conclusions de l'augmentation considérable que vient de connaitre l'effort de recherche américain durant la guerre pour appeler à l'édification d'un système d'information scientifique à la hauteur de cet effort, c'est-à-dire mécanisé, manifeste bien cette relation. Dans ce contexte, et à partir du lien théorique originel entre système d'information scientifique et technologies de l'information et de la communication, il est tentant d'établir un lien direct de cause à effet et d'évoquer le développement technique comme une réponse univoque à des besoins engendrés par l'évolution de la recherche scientifique. Une étude un peu attentive de cette histoire montre que les interactions entre l'un et l'autre sont au contraire multiples et diverses.

Le premier type trouve son modèle en 1968, au moment où est constitué le premier groupe de travail qui conçoit et met en place le premier embryon de réseau d'ordinateurs interconnectés et le protocole informatique qui permet d'y parvenir. Les présentations habituelles de cette histoire qui la résument sous la forme d'une commande de l'armée américaine, sont simplificatrices. En vérité, la plupart des témoignages disponibles sur ce travail [HAU 97] confirment la réalité d'un cadre de recherche relativement libre. Au niveau supérieur, les efforts déployés par son inspirateur, J.C.R. Licklider, pour développer de nouvelles techniques de communication par ordinateurs, manifestaient l'émergence d'une idéologie générale de la communication depuis le début des années 50, étudiée et mise ne lumière par Philippe Breton[BRE 04] et dont il était un des représentants les plus visibles via des publications prophétiques célèbres [LIC 68]. Ce qui est véritablement intéressant pourtant, c'est que constitué sur un mandat flou, le groupe qui travaille sous sa direction était dès lors incité à inventer non seulement une nouvelle technologie, mais aussi à en imaginer les usages. II a donc commencé à utiliser le dispositif qu'il était en train de mettre en place dans le cadre de pratiques d'informations scientifiques et techniques. Patrice Flichy a bien analysé ce développement en montrant à quel point il était symptomatique du mode de fonctionnement d'un idéal de cité scientifique, fondé sur la constitution de "collèges invisibles " conçus comme des communautés d'égaux, la coopération plutôt que la compétition et la coupure par rapport au reste de la société [FLI 01]. De manière significative, le fonctionnement particulier de ce groupe, basé comme on l'a vu sur un mode d'interrelation propre aux milieux scientifiques, s'est ensuite constitué en quelque sorte en modèle lorsqu'il s'est agi de mettre en place des systèmes de régulation du réseau via la constitution de groupes de travail techniques, comme l'Internet Engineering Task Force, d'organismes régulateurs, comme l'Internet Corporation for Assigned Names and Numbers [MUE 04], voire des institutions publiques ou parapubliques dans différents pays [MAR 03]. Le problème est qu'à partir du moment où le réseau s'ouvre et s'étend, il devient un enjeu important pour d'autres milieux socio-professionnels que les milieux scientifiques. Les organismes régulateurs se trouvent alors au centre de conflits opposant plusieurs modèles de communication et de régulation, parmi lesquels le modèle scientifique, basée sur des échanges libres et réciproques entre égaux, n'est qu'un parmi d'autres [MOU 02].

Par ailleurs, il faut évoquer le rôle majeur que joue une discipline particulière dans le développement des TIC en général, mais aussi l'usage de ces technologies dans l'ensemble des autres disciplines scientifiques. En 1989, Tim Berners-Lee propose au CERN qui l'emploie, de mettre en place un nouveau système documentaire permettant de répondre aux contraintes qu'imposent le développement de très gros projets de recherche comme ceux qu'est conduit à assumer l'institut de recherches nucléaires. Le document qu'il écrit, "Information management, a proposal » [BER 89], annonce le World Wide Web, tel qu'il émergera de fait deux ans plus tard, 
reposant en particulier sur le principe de l'hypertexte appliqué à Internet. On n'en déduit pas que le CERN a inventé le Web en guise de réponse à son problème documentaire particulier. Bien d'autres ont préfiguré avant lui ce que pourrait être un système hypertextuel, pas nécessairement dans un contexte purement académique. On remarquera tout de même que c'est bien ce contexte d'usage qui a provoqué l'étincelle conduisant à définir l'hypertexte comme un système documentaire particulier, appliqué par la suite à d'autres contextes. Au delà d'ailleurs de l'hagiographie qui pointe les projecteurs de la célébrité sur un seul homme, on ne manquera pas de remarquer le rôle considérable que joue son institution dans ce développement. Le très important centre de recherche en physique des particules qu'est le CERN doit en permanence depuis sa création, et du fait même de son gigantisme, résoudre des questions de gestion de l'information scientifique au sein de sa propre communauté de chercheurs, ce qui rend naturel l'intérêt qu'il peut porter à l'utilisation des TIC dans le contexte de la communication scientifique [PIG 04]. Ce qui est plus inattendu, c'est que l'innovation technique que constitue le système proposé par Berners-Lee et Cailliaut est immédiatement sortie du contexte de communication scientifique pour laquelle il a été conçu et s'est diffusé au point d'avoir de conséquences très importantes dans l'ensemble des secteurs d'activités.

De l'autre côté de l'Atlantique, en 1991, un chercheur du laboratoire de physique nucléaire encore, à Los Alamos, Paul Ginsparg, met en place un serveur permettant à l'ensemble de sa communauté de recherche d'échanger facilement et très rapidement les articles en pré-publication sur lesquels ils sont en train de travailler (pre-prints). Ce serveur, baptisé Arxiv, s'inscrit, de l'aveu même de son concepteur [GIN 94], non pas en rupture mais en continuité avec une pratique ancienne de communication scientifique propre à sa communauté, basée sur l'échange intensif et ouvert de littérature grise. Au cours des trente dernières années cette communauté s'est manifestement saisie de tout moyen technique lui permettant d'accélérer et d'intensifier ses échanges, le serveur centralisé venant finalement constituer un démultiplicateur de plus dans cette pratique de "communication scientifique directe », comme on l'évoque aujourd'hui. Pour un nombre non négligeable de disciplines scientifiques, Arxiv constitue un véritable modèle faisant l'objet d'une réinterprétation dans leur propre contexte de communication. Modélisé sous le terme générique $d$ ' " archive ouverte ", elle est identifié à la fois par les milieux de la recherche, mais aussi par les bibliothèques scientifiques (à l'origine de l'Open Archive Initiative en 1999) et finalement par les institutions de recherche elles-mêmes (Déclaration de Berlin en 2003), comme le moyen le plus efficace de trouver une solution à la fameuse " crise des périodiques scientifiques " [GUE 01] qui affecte la communication scientifique et dont une des origines paradoxales doit être recherchée dans cette explosion du nombre de publications qu'évoquait Bush au sortir de la guerre. De la mise en place d'un seul serveur d'échange mettant à disposition des pré-publications en physique des hautes énergies uniquement, nous en sommes aujourd'hui à l'existence de plusieurs dizaines d'archives ouvertes dans nombre de disciplines [HAL 06]. Celles-ci donnent aussi accès à des milliers de postpublications (le terme d'e-prints, employé depuis, désigne aussi bien pré que post-publications). Sous l'influence non pas des technologies de l'information et de la communication, mais plus sûrement de l'usage qu'en font certaines communautés scientifiques en réaction aux nouvelles conditions de publication qui leur sont imposées, les pratiques de publication et de communication scientifique sont en train de changer considérablement.

D'autres cas, plus récents et moins analysés, pourraient enfin être évoqués, à l'instar du logiciel libre. Ce mouvement, qui s'étend à partir de 1984 sous l'impulsion d'un chercheur du Massachusetts Intitute of Technology, porte et reformule politiquement des normes clairement issues du monde scientifique. Qualifié de «communiste» par ses détracteurs (et quelques-uns de ses partisans), le logiciel libre exprime bien plutôt des valeurs " communalistes », c'est-à-dire de mise en commun et de partage des connaissances qui sont constitutives de la recherche scientifique. Ce qui est intéressant, c'est la manière dont ce mouvement, dont l'origine peut être identifiée dans des pratiques de partage du code informatique entre programmeurs au sein des milieux académiques, a agrégé de multiples manières un public bien plus large autour de valeurs politiques partagées. Ainsi a-t-il construit d'importantes communautés d'utilisateurs autour de logiciels particuliers [AUR 05] au sein desquelles se diffusent, avec différentes sortes de reformulation, des éléments propres à son 
milieu d'origine. On notera d'ailleurs qu'en retour, le logiciel libre a historiquement joué un rôle important comme modèle et exemple pour le développement des archives ouvertes déjà évoquées [MOU 05].

Pour une bonne part, les TIC doivent le développement sans précédent qu'elles ont connu au cours des dernières décennies à l'émergence de nouvelles questions posées par l'évolution de l'ensemble du champ scientifique et de ses pratiques de communication. Ces processus sont particulièrement manifestes dans le domaine de l'édition électronique scientifique, même s'ils restent plus difficiles pour les formes canoniques de diffusion des résultats de la recherche que pour des objets éditoriaux en cours d'émergence, fruits d'emprunts à des contextes de publication non académiques. Le cas des sciences humaines et sociales et la manière dont elles ont produit des résultats différenciés selon les formes éditoriales en constitue un exemple flagrant.

\section{De la confrontation à l'appropriation évolutive des modèles}

A bien des égards, la relation qu'entretiennent les sciences humaines avec Internet prend les traits d'une confrontation de modèles. Très rapidement, le monde de la recherche et de l'enseignement francophones a souhaité prendre pied sur ce nouveau continent. D'une façon qui paraissait tout à fait logique, il a d'abord pensé en général à y transposer l'objet le plus noble qui soit, la monographie. C'est ainsi qu'en 2000 Jean-Marie Tremblay a créé les Classiques des sciences sociales ${ }^{2}$, qui consiste à mettre en place une bibliothèque contenant les ouvrages de Gustave Le Bon, Frédéric Le Play, François Simiand et des dizaines d'autres auteurs classiques en sciences sociales (en mai 2006, le corpus approche les 2000 titres). La plateforme Cyberdocs permet à l'Université Lumière Lyon 2 de diffuser 754 thèses consultables en ligne ${ }^{3}$. La plateforme canadienne Érudit ${ }^{4}$ ou ENS-Éditions à Lyon ${ }^{5}$ publient également quelques ouvrages électroniques. La taille, la qualité formelle et technologique de ces initiatives est variable, mais quelques tendances semblent se dégager. Deux stratégies différentes ont été adoptées, parfois de façon exclusive. D'une part, stratégie du téléchargement d'un fichier, objet clos, auto-suffisant, prêt à l'impression domestique, mais déconnecté du maillage de plus en plus dense que constitue le Web. Stratégie du site web, d'autre part, objet qui s'accompagne d'une table des matières et de notes hypertextuelles, consultable en ligne, moins aisément imprimable. En général, cette deuxième stratégie favorise l'indexation par les moteurs de recherche généralistes, donc la visibilité des documents au-delà du public universitaire. Les vertus de telles démarches sont considérables : même si les statistiques de fréquentation ne sont pas toujours connues, la mise à disposition mondiale de telles ressources permet de profiter des qualités de circulation du support électronique. La recherche d'occurrences précises est également facilitée, si ce n'est sur le corpus total, au moins sur un ouvrage particulier. Pour l'essentiel, la forme et l'écriture monographiques sont conservées, voire cultivées. L'héritage de la forme papier n'est pas remise en cause, la finalité explicite d'un certain nombre de livres électroniques étant d'être imprimés, pour tout ou partie.

Sans évoquer les projets d'encre électronique [WIL 03] [KRE 06], plusieurs projets de monographies électroniques ont tenté d'infléchir le modèle initial pour l'adapter d'avantage aux qualités de l'électronique. Tous imposent, peu ou prou, de repenser la forme canonique de l'ouvrage pour l'adapter à un nouveau support. La proposition de l'historien du livre Robert Darnton a été très remarquée [DAR 99]. Elle s'appuie sur la métaphore de la pyramide, au sommet de laquelle se situerait une synthèse, appelée à être diffusée sur papier. Cette pyramide se déplierait ensuite en cinq niveaux supplémentaires (argumentation détaillée, documents de travail, considérations théoriques ou historiographiques, propositions pédagogiques, échanges et débats avec les lecteurs). L'apport principal de la démarche consiste à prévoir une gamme d'usages variés, mais surtout, nous semble-t-il, d'ouvrir le champ des possibles en rappelant que la lecture linéaire, de la première à la

Les Classiques des sciences sociales : http://classiques.uqac.ca/ . Nous remercions Jean-Marie Tremblay pour les réponses qu'il a bien voulu apporter à nos questions concernant son projet.

Université Lyon 2, les thèses électroniques : http://demeter.univ-lyon2.fr:8080/sdx/theses/

Érudit : http://www.erudit.org

ÉNS éditions : $\underline{\text { http://editions.ens-lsh.fr/ }}$ 
dernière page d'un ouvrage, n'est qu'une des modalités de lecture sur papier; et que le support électronique recèle intrinsèquement un potentiel de diversifications des usages de l'écrit encore plus grand. Cependant, Robert Darnton insiste sur le fait qu'il ne s'agit pas de connecter le livre à de grandes bases de données, mais bien de structurer et de rédiger autrement le propos scientifique luimême. Les projets de monographie électronique défendus par Valentine Roux [ROU 04] [ROU 04bis] relèvent d'une approche encore plus formelle, en partant du constat de l'inflation du nombre de publications produites par la recherche, et proposant eux aussi une écriture nouvelle, une réécriture logiciste s'appuyant sur les moyens multimédia. Ce travail de réécriture constitue sans doute un obstacle à la généralisation de tels projets. La contrainte que l'on pourrait qualifier d'« oulipienne », c'est-à-dire formelle, du nombre de pages du livre imposait de fait une économie de l'écriture qui, malgré les frustrations induites, constituait également une économie temporelle de l'écriture. Or, les contraintes du marché des carrières académique ne sont pas négligeables et les chercheurs disposent d'un capital de temps limité pour publier les résultats de leurs recherches. Dès lors, le principal frein au déploiement d'une « monographie électronique idéale » est bien la prise en compte du temps nécessaire à sa rédaction, ou plutôt à ses écritures. De fait, en dehors de Cyberthèse/Cyberdocs et des Classiques des sciences sociales, aucun mouvement de fond ne semble s'être enclenché, qui pourrait augurer d'une tendance émergente. On peut cependant accorder une place à part à l'édition de sources, notamment dans le domaine archéologique et paléographique. Le coût important de la publication de matériaux sur support papier, pour un lectorat extrêmement spécialisé, combiné à la complexité du travail d'édition savante, ont fortement incité les communautés scientifiques concernées à explorer les voies de l'édition électronique. L'École nationale des Chartes en est un exemple remarquable, avec la réalisation de plusieurs volumes entièrement conçus et diffusés de cette manière. Une telle expérience, qui repose sur une structuration forte de l'information en XML (Text Encoding Initiative), requiert une haute technicité du travail d'édition et une exploration des possibilités de navigation et de mise en relations permises par l'hypertexte et l'écran ${ }^{6}$.

Les périodiques ne présentent pas le même type de difficulté [DAC 05]. L'unité documentaire qui doit être traitée n'est plus l'ouvrage (un ensemble de 500000 à plusieurs millions de signes), mais un article (dix à douze fois moins). Dès lors, les problèmes de structuration du texte, les technologies de mise en ligne, les contraintes ergonomiques, les questions d'indexation ont trouvé des réponses plus rapidement et avec plus de facilité. C'est donc historiquement cette forme de publication qui a vu se développer le premières initiatives importantes de mise en ligne : Muse ${ }^{7}$, Highwire $^{8}$, Jstor ${ }^{9}$ pour le monde anglophone, Érudit, Revues.org ${ }^{10}{ }^{\text {et }}$ Cairn ${ }^{11}$ pour les publications francophones. Mais traditionnellement, les publications périodiques ne bénéficient pas du même prestige que les monographies en sciences humaines et sociales. On peut se demander si ce coup de projecteur porté sur les revues n'aboutit pas, in fine, à rapprocher les SHS de modèles de communication qui prévalent ailleurs. On peut émettre l'hypothèse que les archives ouvertes, qui concernent pour l'essentiel, comme on l'a dit, les articles, joue un rôle non négligeable dans cette évolution. Des disciplines comme l'économie ont adopté très tôt ce type de dispositif, et l'archive ouverte REPEC, ouverte dès 1997, en est un bon exemple.

On aurait tort, cependant, de concentrer l'attention sur la publication canonique. C'est en amont de la publication que le numérique a le plus de chances de déplacer les lignes et de favoriser l'innovation. La première nouveauté, désormais quasiment transparente, le courrier électronique, est très rapidement devenue une instrument d'usage quotidien, voire intensif, même s'il est manifestement rarement expert. A cet usage liant les individus entre eux s'est greffée la diffusion, également rapide, des lettres d'information et, en parallèle, des listes de discussion, qu'elles soient internes à un groupe, semi-publiques ou totalement publiques. Leur nombre est tel qu'il n'est pas

\footnotetext{
Le graduel de Bellelay : $\underline{\text { http://bellelay.enc.sorbonne.fr/ }}$

Project Muse, scholarly journals online : http://muse.jhu.edu/

Highwire Press : http://highwire.stanford.edu/

Jstor, the scholarly journal archive : http://www.jstor.org/

10 Revues.org, Edition électronique scientifique : http://www.revues.org

1 Cairn, chercher, repérer, avancer : $\underline{\text { http://www.cairn.info }}$
} 
possible de les citer, en dehors du célèbre groupement de listes $\mathrm{H}-{ }^{-n e t}{ }^{12}$ et de ce qui constitue probablement la liste la plus ancienne et la plus importante en sciences humaines et sociales, Biblio$\mathrm{fr}^{13}$. Si on a pu croire, à leurs débuts, que les listes favoriseraient le débat et l'échange, il a fallu rapidement se rendre à l'évidence : elles sont rarement propices aux débats théoriques mais très efficace pour le partage du savoir-faire et, plus encore, pour la diffusion de l'actualité de groupes ou d'individus. Dans leur prolongement ont émergé des pages d'actualités publiées par les établissements d'enseignement et de recherche, puis des sites d'actualités scientifiques ${ }^{14}$. Ces multiples canaux de diffusion de l'actualité de la recherche ont contribué à une plus grande ouverture de l'accès à la vie scientifique [DAC 00], permettant notamment à de jeunes chercheurs de moins dépendre de leur environnement immédiat pour saisir les tendances nationales et internationales de leur spécialité, de leur discipline et des disciplines proches. Si ce type d'objet éditorial fonctionne bien, c'est notamment en raison de son faible caractère polémique : en général, la publicité des événements scientifiques est souhaitée.

Il en va autrement pour les écrits qui précèdent, accompagnent ou suivent la publication, c'est-à-dire la littérature grise, les notes préparatoires, les carnets d'enquête, les documents de synthèse. Une partie de ces documents peut aujourd'hui être publiée par l'intermédiaire d'un objet éditorial né, il y a plusieurs années, dans un contexte non académique : le blog. S'il est bien difficile, de donner une origine exacte à cette forme éditoriale, comme souvent, on en situe cependant les débuts au milieu des années 90, avec les blogs de Justin Hall et Dave Winner, l'un journaliste, l'autre développeur américains. Ce qui caractérise les premiers blogs, et qui constituera une marque distinctive de ce genre, c'est un mode d'énonciation particulièrement subjectif, à l'opposé de celui qui est attendu, par exemple, dans une publication académique. Et de fait, la popularité grandissante de ce mode de publication a accompagné le mouvement de démocratisation du web, à partir de la fin des années 90, c'est-à-dire le moment précisément où Internet « sort des campus » et touche bun public bien plus large que les milieux académiques.

Caractérisé par une mise en ligne simple et rapide de billets parfois courts, classés dans l'ordre chronologique de leur publication et ouverts aux commentaires du lectorat, le blog, véritable phénomène de masse depuis 2004, gagne peu à peu la sphère universitaire, même si les usages sont encore peu nombreux et marginaux d'un point de vue institutionnel. L'acculturation de la communauté scientifique à ce modèle qu'elle n'a pas suscité produit au moins quatre types de blogs différents. Tout d'abord, émerge une évolution presque banale des sites personnels ou d'équipes de recherche des débuts de l'Internet, comportant principalement un CV et une bibliographie. Le principal intérêt est ici de faciliter la mise à jour du site, ce qui laisse espérer que l'obsolescence des contenus ne sera pas aussi chronique que dans la génération précédente de sites. La croissance de ce type de blog semble inexorable et propice à la circulation de l'information ${ }^{15}$. Le deuxième type de blog, alimenté par des enseignants, trouve son utilité dans le cadre de l'amphithéâtre ou du séminaire, comme enrichissement de la relation pédagogique ${ }^{16}$. Le troisième s'adresse à un plus large public et tente de répondre à une demande sociale. A l'heure où des voix posent la question de l'utilité des sciences humaines, notamment en ce qui concerne le financement de la recherche par l'Etat, les blogs peuvent constituer de véritables ambassadeurs pour ces disciplines, montrant la pertinence de leurs objets d'études, l'intérêt des savoirs qu'elles constituent pour l'enrichissement du débat public. Le carnet de recherches, quatrième type, est sans aucun doute aujourd'hui le moins bien représenté. Publié en temps réel ou après un prudent délai de restriction, il serait le lieu où consigner ses dernières trouvailles, expérimenter de nouvelles pistes, forger, tester et commenter formulations et concepts. Associé à des commentaires ouverts au lectorat, il constitue sans doute une forme de séminaire électronique. Encore embryonnaire, le carnet de recherche sera-t-il un

\footnotetext{
H-net, humanities and social sciences online : http://www.h-net.org/

Biblio-fr, Bibliothécaires, documentalistes et documents numériques : $\mathrm{http}: / /$ listes.cru.fr/sympa/info/biblio-fr

Calenda, l'agenda des sciences sociales : http://calenda.revues.org

Urfistinfo, Actualité des Sciences de l'Information : http://urfistinfo.blogs.com/urfist info/, ViteVu, le blog de la société française de photographie : http://www.societefrancaisedephotographie.fr/blog

16 Technologies du langage, Actualités, commentaires, réflexions : http://aixtal.blogspot.com/ et Réseaux, etc. : http://lemercier.ouvaton.org/
} 
nouveau « gueuloir de Flaubert », ou plutôt la version numérique du carnet de terrain ${ }^{17}$, ou encore un ensemble informel, constitué de notes mêlant dans un joyeux désordre les informations, les concepts, les références et les commentaires ? Quoi qu'il en soit, il est manifeste que la typologie proposée est essentiellement formelle et que les sites concernés sont souvent des versions hybrides de plusieurs formes mises en évidence ${ }^{18}$, voire de formes totalement nouvelles, comme le blog de revue ${ }^{19}$.

Déployer l'économie de la production de formes éditoriales et son déséquilibre en faveur de ce qui paraît a priori le moins rentable d'un point de vue scientifique, revient à évoquer la question de la rétribution symbolique de la création d'un blog de chercheur. Les freins sont nombreux. Le premier est le risque de sanctions académiques [CLO 05] à l'égard de son auteur. On sait que la tradition du site personnel n'a quasiment jamais pris en sciences humaines et sociales, en France, en raison d'un préjugé a priori négatif affecté à la mise en avant de soi. Les blogs de chercheur posent le même problème, et il ne faut pas s'étonner que les premiers blogs francophones en sciences humaines et sociales concernent des disciplines proches des sciences dures, de chercheurs d'Amérique du nord ${ }^{20}$, de français exilés ${ }^{21}$ ou d'auteurs non universitaires ${ }^{22}$. Le second est celui de la diffusion prématurée d'informations, favorisant l'emprunt voire le plagiat d'idées nouvelles avant que leur inventeur aie pu en faire son miel. Cet argument explique la rareté des blogs de recherche, au profit des blogs pédagogiques ou de vulgarisation. Mais il peut rapidement se renverser, et passer au rang des avantages. En effet, la publication en ligne, sur un blog de chercheur, d'une piste de recherche nouvelle ne permet-elle pas d'en assurer rapidement et efficacement la publicité, donc d'en établir sans discussion possible la paternité en même temps que de permettre l'évaluation collective de sa validité au sein de l'ensemble de la communauté scientifique concernée ? Cette perspective dessine peut-être un trait spécifique aux blogs de chercheurs. Par ailleurs ils permettent de toute évidence une identification plus facile du chercheur dans sa spécialité, ce qui est particulièrement crucial pour les plus jeunes d'entre eux, mais aussi pour tous ceux qui souhaitent évoluer rapidement vers de nouveaux objets d'étude. Ainsi, le blog de chercheur pourrait être un des facteurs d'oxygénation du milieu de la recherche, en favorisant la circulation des idées et en compensant certains des effets négatifs du fonctionnement en microcosme. Enfin, le dernier avantage de ce type de blogs, surtout lorsqu'il est collectif, consiste probablement en ce qu'il constitue un outil facilitant la structuration de champs de recherche émergents, facilitant leur visibilité pour les tutelles et peut-être ensuite, le déclenchement de financements permettant une organisation de plus grande ampleur. A l'issue de ce rapide tour d'horizon, force est de constater que, si les freins restent nombreux, c'est essentiellement parce que des questions de fond ne sont pas encore éclaircies, même si ceux qui se sont jetés à l'eau semblent plutôt satisfaits de leur expérience [GUN 06] [ROL 06]. La parole des chercheurs sera-telle employée pour mesurer une mesure d'impact des revues, des chercheurs, des unités de recherche, des établissements ? Il semble que, malgré le risque d'instrumentalisation et les biais inévitables d'une telle approche, les avantages soient plus importants que les inconvénients. En effet, on peut espérer que, couplées aux dépôts d'archives ouvertes ${ }^{23}$ et aux portails de revues, les mesures d'impact prenant en compte les blogs de chercheurs permettront de dresser un panorama plus juste et plus représentatif de la science en train de se faire, alors que les mesures traditionnelles, exclusivement calibrées sur le support papier, donnent une représentation décalée dans le temps et ont tendance à laisser les autorités scientifiques établies s'auto-légitimer [HEN 04]

17 Philippes 2004, éphéméride d'une campagne de fouilles en Macédoine : http://philippes2004.blogspot.com/

18 Baptiste Coulmont $:$ http://coulmont.com/blog/, Blogo Numericus : http://homo-numericus.net/blog/, Archives de la Recherche en Histoire Visuelle : http://www.arhv.lhivic.org/

19 Tour de Toile, Le blog du BBF, Veille sur l'actualité des bibliothèques et des sciences de l'information : http://blogbbf.enssib.fr/

20 Recherches en littérature contemporaine : http://carnets.contemporain.info/audet/

${ }_{21}$ L'Economie sans tabou, Les commentaires d'un économiste sur l'actualité et les problèmes économiques : http://bsalanie.blogs.com , Bloc-notes de Jean-Michel Salaün, Repérage de données sur l'économie des documents dans un environnement numérique $: \underline{\mathrm{http}}$ ://grds04.ebsi.umontreal.ca/jms/

22 Boire et manger, quelle Histoire! Le blog d'une historienne de l'alimentation : http://segolenelefevreampelogos.hautetfort.com/histoire des aliments/

23 HAL SHS, Archive ouverte en sciences de l'homme et de la société : http://halshs.ccsd.cnrs.fr 
[GUN 04].

Les blogs de chercheurs semblent avoir acquis une visibilité dépassant le public habituel de leurs auteurs. Mais ils en subissent les contre-effets, sous la forme d'une confrontation entre les modèles académiques, dont ils procèdent, et des modèles issus de la société, dont ils s'inspirent. Les "nouveaux modèles" de structuration de l'information, popularisés sous le nom de "Web 2.0" [ORE 05], mettent en cause le modèle universitaire caractérisé par le tryptique auteur-éditeur-lecteur. En effet, ils en recomposent les figures et redessinent partiellement les frontières, notamment à travers les pratiques de " co-marquage » [HAM 05], de validation a posteriori [POU 06] et d'« écritures collectives » (notamment de type Wiki, mais pas seulement). En rapprochant les univers du profane et du savant, ce processus enrichit la société mais embarrasse et, parfois, déstabilise l'identité des chercheurs et des institutions qui les structurent. La controverse qui a accompagné la spectaculaire croissance de Wikipedia $^{24}$ semble représentative des problèmes engendrés par l'abus des métaphores et par l'utilisation d'un vocabulaire devenu obsolète pour décrire des formes éditoriales nouvelles. Wikipedia ne peut qu'attirer l'ire des spécialistes en tous genres si on laisse perdurer l'idée qu'il s'agit d'une encyclopédie au sens traditionnel du terme [KLE 05]. Les porteurs du projet Jurispedia l'ont bien compris, qui s'inspirent d'un modèle efficace, mais le construisent en tant qu'objet distinct de l'encyclopédie collaborative phare, conçu et géré par des universitaires ${ }^{25}$. De même, certaines communautés académiques ont su s'inspirer de dispositifs à destination du grand public $^{26}$, pour proposer des outils spécialisés ${ }^{27}$. A travers un ensemble d'ajustements, d'adaptations et d'inventions difficiles à prévoir dans le détail, ce processus d'interaction entre sciences et société semble amené à se poursuivre.

\section{Conclusion}

Comme espace et vecteur d'interactions entre sciences de la nature, société et sciences humaines et sociales, Internet constitue un phénomène majeur, dont il est encore difficile de dresser le bilan définitif. Cependant, apparaissent avec évidence des phénomènes d'enrichissements réciproques mettant en jeu technologies, formes éditoriales et modalités d'échange. Fonctionnant comme une sorte d'alternateur, le processus à l'oeuvre est multiple et fortement dépendant des acteurs. Il redéfinit sans cesse le statut, la légitimité et le rôle de chacun. Son caractère imprévisible est souvent vécu comme un travail de sape affaiblissant les piliers sur lesquels se construisent les identités professionnelles, en particulier parmi ceux des acteurs qui dominent leur champ et craignent de voir leurs positions remises en cause. Pour les sciences humaines et sociales, dont le statut est historiquement au centre de débats récurrents, ce bouleversement est parfois vécu de manière dramatique. Au-delà des prises de positions extrêmes, il doit être l'occasion, nous semble-til, de s'interroger sur les conditions contemporaines de production et de circulation des savoirs, en en examinant toutes les dimensions, y compris celles qui concernent les technologies et leurs usages.

\section{Bibliographie}

[BER 89] Tim Berners-Lee. Information Management: A Proposal, Genève, CERN DD/OC, March 1989. (partout)[BRE 04] Breton, Philippe, L'utopie de la communication, Paris, La Découverte, 2004

[BUS 45] Bush, Vanevar, «As we may think », The Atlantic Monthly, Washington D. C., Atlantic Media, Volume 176, No. 1, p. 101-108, juillet 1945

[CLO 05] Cloutier, Jean-Pierre, «Blogues : Universitaires à risque? », Jean-Pierre Cloutier, Le blog, Montréal, Les Editions Cybérie, disponible en ligne : [

Wikipedia : http://www.wikipedia.org

Jurispedia : http://www.jurispedia.org/

Delicious : $\underline{\text { http://del.icio.us }}$

Connotea, a free online reference management service for researchers and clinicians : $\underline{\mathrm{http}} / / / \mathrm{www}$.connotea.org/ et

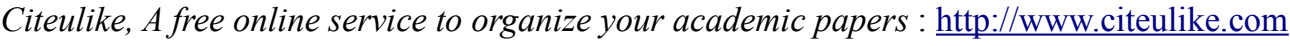


http://cyberie.qc.ca/jpc/2005/10/blogues-universitaires-risque.html [Réf. du 31/03/06], 13 octobre 2005

[DAC 00] Dacos, Marin, « Un historien au pays du Minitel. Le Web et l'histoire en France à la fin de l'année $2000 »$, Journal of the association for history and computing, vol. III, n³,Greencastle, AAHC , 2000

[DAC 05] Dacos, Marin, Murzilli Nancy, Secondat de Montesquieu, Inès, « Développer les usages en ligne : de l'éditeur électronique au lecteur. Le cas de Revues.org », in Les historiens, leurs revues et Internet. (France, Espagne,Italie) (2005), p. 131-148, Paris, Publibook, 2005

[DAR 99] Robert Darnton, "The New Age of the book», The New York Review of Books, New York, NYREV Inc., vol. 45, $\mathrm{n}^{\circ} 55$, disponible en ligne : [http://www.nybooks.com/articles/546] [Réf. du. 31/03/06], 18 mars 1999

[FLI 01] Flichy, Patrice, L'imaginaire d'Internet, Paris, La Découverte, 2001

[GIN 94] Ginsparg, Paul, « First Steps Towards Electronic Research Communication », Computers in Physics, Maryland, American Institut of Physics, v.8, n.4, p. 390-396, 1994

[GUE 01] Guédon, Jean-Claude, «In Oldenburg's Long Shadow: Librarians, Research Scientists, Publishers, and the Control of Scientific Publishing ", ARL Proceedings, Washington D. C., Association of Research Librairies, $\mathrm{n}^{\circ} 138$, disponible en ligne : [http://www.arl.org/arl/proceedings/138/guedon.html] [Réf. du 31/03/06], Mai 2001

[GUN 04] Gunthert, André, "La punition des revues », Revue d'Etudes Photographiques, Paris, Société d'Etudes Photographiques, $\mathrm{n}^{\circ} 15$, disponible en ligne : [http://etudesphotographiques.revues.org/document392.html] [Réf. du 31/03/06], novembre 2004 [GUN 06] Gunthert, André, "Arhv, premier bilan », Actualités de la recherche en histoire visuelle, Paris, Laboratoire d'Histoire Visuelle Contemporaine, disponible en ligne : [http://www.arhv.lhivic.org/index.php/2006/05/23/180-arhv-premier-bilan], [Réf. du 31/05/06], 23 mai 2006

[HAM 05] Hammond, Tony, Hannay, Timo, Lund, Ben et Scott, Joanna, « Social Bookmarking Tools (I). A General Review. », D-Lib Magazine, vol. 11, $\mathrm{n}^{\circ} 4$, disponible en ligne : [http://www.dlib.org/dlib/april05/hammond/04hammond.html], [Réf. du 31/05/06], Avril 2005

[HAU 97] Hauben Michael, Hauben Ronda, Netizens: On the History and Impact of Usenet and the Internet, Wiley, IEEE Computer Society Press, disponible en ligne : [http://www.columbia.edu/ hauben/netbook/], [Réf. Du 31/05/06], April 1997

[HEN 04] Henriot, Christian (dir), Sciences de l'homme et de la société, Numéro spécial, "Les revues en sciences humaines et sociales », Paris, CNRS, n64, mai 2004

[KLE 05] Klein, Arnaud, "Wikipédia et la légitimité de la construction collective du savoir sur internet », Internetactu, disponible en ligne : [http://www.internetactu.net/?p=5941], [Réf. du 31/03/2006], 25 mai 2005

[KRE 06] Krebs, Constance, «Du livre électronique à l'encre électronique. Nouveau papier, nouveau livre?», Bulletin des bibliothèques de France, Lyon, ENSSIB,t.51, n4, p. 38-41, disponible en ligne : [http://bbf.enssib.fr], [Réf. du 31/07/2006], 2006

[LIC 68] Licklider, J.C.R. and Taylor, R.W., « The computer as Communication Device », Sci. Tech., p. 21-31, Avril 1968

[MAR 03] Marzouki M., Méadel C., 2003, «Gouvernance d'Internet, collectifs électroniques, régulation, production de normes», Paris, CNRS, rapport de fin d'action, 2003

[MOU 02] Mounier, Pierre, Les Maîtres du réseau, les enjeux politiques d'Internet, Paris, La Découverte, 2002

[MOU 05] Mounier, Pierre, « Technologies libres et société », La Revue Nouvelle, Bruxelles, n6-7, juin-juillet 2005

[MUE 04] Mueller, Milton, Ruling the Root: Internet Governance and the Taming of Cyberspace, Cambridge, MIT Press, 2004

[ORE 05] O'Reilly, Tim, «What Is Web 2.0. Design Patterns and Business Models for the Next Generation of Software », O'Reilly, Sebastopol (Ca), O'Reilly Media, disponible en ligne : [http://www.oreillynet.com/pub/a/oreilly/tim/news/2005/09/30/what-is-web-20.html], [Réf. Du 31/03/2006], 30 septembre 2005 
[POU 06] Poupeau, Gautier, «Blogs et wikis. Quand le web s'approprie la société de l'information », Bulletin des bibliothèques de France, Lyon, ENSSIB, t.51, n॰3, p. 29-37, disponible en ligne : [http://bbf.enssib.fr], [Réf. du 31/07/06], 2006

[PIG 04] Pignard-Cheynel, « Nathalie, La communication des sciences sur Internet, Stratégies et pratiques », Grenoble, Université Grenoble 3, Thèse de doctorat, septembre 2004

[ROL 06] Rolin, Frédéric, "100ème note, un bilan, des perspectives », Le blog de Frédéric Rolin, Paris, Blogspirit, disponible en ligne : [http://frederic-rolin.blogspirit.com/archive/2006/05/28/100eme-note-un-bilan-des-perspectives.html], [Réf. du 31/05/06], 28 mai 2006

[ROU 04] Roux, Valentine, avec la participation de Philippe Blasco 2004, « Faciliter la consultation de textes scientifiques. Nouvelles pratiques éditoriales ", Hermès, Critique de la raison numérique, n³9, p. 151-159, Paris, CNRS éditions, 2004.

[ROU 04bis] Gardin, Jean-CLaude, et Roux, Valentine, « The Arkeotek project: a european network of knowledge bases in the archaeology of techniques ", Archeologia e Calcolatori, $\mathrm{n}^{\circ} 15, \mathrm{p}$. 25-40, Firenze, Edigiglio, 2004

[SAU 06] Saunier, Pierre-Yves, « Des listes électroniques : pour quoi, pour qui ? Notes sur les historiens français et les communautés en ligne », in Rygiel, Philippe, Noiret, Serge, (dirs.), Les historiens, leurs revues et Internet (France, Espagne, Italie), Paris, Publibook, p.115-128, 2005

[SCH 05] Schiele, Bernard, «Publiciser la science ! Pour quoi faire ?» in Paillard, Isabelle (dir.), La Publicisation de la science, Grenoble, PUG, 2005

[WIL 03] Wilson, Ruth, « Displaying Digital Information on Paper-like Devices », Rapport pour le JISC, disponible en ligne : [http://www.jisc.ac.uk/index.cfm?name=techwatch_report_0301], [Réf. du 31/05/06], janvier 2003 